\title{
Autologous platelet-rich plasma optimizes endometrial thickness and pregnancy outcomes in women with refractory thin endometrium of varied aetiology during fresh and frozen-thawed embryo transfer cycles
}

\author{
Yogita Dogra ${ }^{1}$, Neeta Singh ${ }^{1}$, Perumal Vanamail ${ }^{1}$ \\ ${ }^{1}$ ART Center, Department of Obstetrics and Gynecology, All India Institute of Medical Sciences, Ansari Nagar, \\ New Delhi, India
}

Meeting where work was presented- Abstract (in part) as e-poster at 36th Virtual ESHRE Meeting, July 2020

\begin{abstract}
Objective: To evaluate whether platelet-rich plasma (PRP) optimizes endometrial thickness (EMT) and improves live birth rates (LBR) in women with refractory thin endometrium due to varied aetiology during fresh in vitro fertilization (IVF) and frozen-thawed embryo transfer (FET).
\end{abstract}

Methods: A prospective interventional study was conducted at the ART Centre of a tertiary care academic hospital. Twenty-two infertile women with refractory thin endometrium $(<7 \mathrm{~mm})$ despite standard hormone replacement therapy were assessed. Twenty patients underwent 26 PRP cycles from December 2018 - June 2020 during fresh IVF-ET and FET. Primary endpoint was expansion of EMT and secondary outcomes were implantation rate (IR), clinical pregnancy rate (CPR) and LBR in fresh and FET cycles and aetiology wise.

Results: Mean EMT increased significantly following PRP administration $(p<0.001)$ with average increase of $1.07 \mathrm{~mm}$ and $0.83 \mathrm{~mm}$ after first PRP $(p<0.001)$ during fresh IVF and FET, respectively. CPR, IR and LBR showed no significant difference when compared during fresh vs. FET cycles $(p>0.05)$. PRP led to significant increase in EMT in tuberculosis (TB), diminished ovarian reserve (DOR) and polycystic ovary syndrome (PCOS) $(p<0.001)$. There was no significant difference in CPR, IR and LBR among three aetiological factors $(p>0.05)$. Overall, clinical pregnancy and LBR reached up to $20 \%$ and $25 \%$, respectively. No adverse reactions were reported.

Conclusions: PRP enhances EMT significantly during fresh and FET cycles in thin endometrium associated with $T B, P C O S$ and DOR, thus improving the CPR and LBR in these low prognosis patients.

Keywords: refractory thin endometrium, PRP, fresh IVF, frozen embryo transfer, endometrial thickness

\section{INTRODUCTION}

Endometrium thickness of $<7 \mathrm{~mm}$ on the day of ovulation, or at the day of human chorionic gonadotrophin (HCG) injection in fresh in vitro fertilisation (IVF) cycles or the day of starting progesterone in frozen-thawed embryo transfer (FET) cycles is characterised as thin endometrium (Liu et al., 2019). It had already been substantiated in a large number of studies that endometrial thickness (EMT) and its pattern were independent factors that influenced pregnancy outcomes. Thin endometrium was pondered as an independent adverse prognostic factor for attaining pregnancy in women, whether or not with ovarian stimulation (Zhao et al., 2014; Zhang et al., 2018; Von Wolff et al., 2018; Bu et al., 2019). Thin endometrium has been associated with lower implantation and pregnancy rates, and its incidence rate is about $2.4 \%$ in individuals undergoing assisted reproductive technology (ART) cycles (Mahajan \& Sharma, 2016).

In females with a normal uterine cavity with a persistent thin lining, several modalities have been explored to improve endometrial thickness, including the use of exogenous estrogens, vaginal sildenafil, low-dose aspirin, and granulocyte-colony stimulation factor (Weckstein et al., 1997; Sher \& Fisch, 2002; Chen et al., 2006; Gleicher et al., 2013). Still, a large fraction of women with thin endometrium remains noncompliant to such therapies. Platelet-rich plasma (PRP) as a novel therapeutic modality in the management of poor responders to HRT has revealed encouraging results in preliminary studies. Instigating effect of PRP has been revealed on endometrial cell proliferation and migration, as well as expression of numerous factors inherently involved in endometrial regeneration and repair (Aghajanova et al., 2018).

PRP has been substantially used in orthopaedics, dental and plastic surgery, diabetic wound healing, and dermatology to sustain tissue growth and repair (Cervelli et al., 2009; Jo et al., 2013; Picard et al., 2015). Its use has been minimally investigated to date in obstetrics and gynaecology practice. Numerous growth factors and cytokines (e.g., VEGF, TGF $\beta$, PDGF, IGF1, FGF, EFG, HGF, CXCL12, and CCL5) amass in platelets and are released at the site of injury or inflammation during platelet activation. Platelet-derived factors are vital for endometrial progenitor cell activity (Gargett et al., 2008), and PDGF isoforms notably prop up endometrial stromal cell proliferation, migration, and contractility (Matsumoto et al., 2005).

Numerous studies have substantiated the effectiveness of local administration of PRP in attaining successful implantation in women with thin endometrium undergoing FET cycles. In contrast, there is a paucity of data on the use of PRP in women with persistent thin endometrium planned for fresh IVF-ET cycles. Results of some studies (Paulson et al., 1990; Hadi et al., 1994) have suggested that endometrial receptivity is noticeably weakened in ovarian stimulation cycles as well, and is one of the rate-limiting factors of IVF (Gonen \& Casper, 1990).

The role of PRP in increasing EMT and improving pregnancy and live birth rates concerning the underlying etiological factor of infertility has not been explored till date. Thin endometrium has been observed in women suffering from PCOS with hyperandrogenemia (Indhavivadhana et al., 2017). Also, women having PCOS undergo multiple ovulation induction cycles with clomiphene before IVF. Clomiphene diminishes endometrial receptivity by impairing endometrial development and uterine blood flow, which 
results in endometrial thinning in $15-50 \%$ of patients. It may lead to subsequent implantation failure and may also induce early pregnancy loss due to luteal phase defect (Hsu et al., 1995; Thessaloniki ESHRE/ASRM-Sponsored PCOS Consensus Workshop Group, 2008). Women with DOR have a reduced cohort of recruitable antral follicles, with resultant decreased estradiol production, in contrast to women with a normal or high ovarian reserve (Scheffer et al., 2003). Estradiol levels have a bearing on endometrial thickness (Kasius et al., 2014). The tubal factor infertility secondary to genital tuberculosis (TB) involves the endometrium in $50-80 \%$ cases (Sharma et al., 2008), making it pale and non-receptive. Whether PRP can increase the endometrial thickness in these underlying etiologies is a subject of interest.

Therefore, the present study was held to appraise the role of PRP during fresh IVF and FET cycles and to explore the benefits of PRP concerning underlying factor of infertility if PRP can be employed in a specific subset of women with thin endometrium with greater success in achieving pregnancy. The primary endpoint was the expansion of EMT during fresh IVF and FET cycle and according to the factor of infertility. The secondary outcomes were implantation rate, clinical pregnancy rate and LBR in fresh and FET cycles and aetiology wise.

\section{MATERIALS AND METHODS}

\section{Study design}

A prospective interventional cohort study was conducted at the Assisted Reproductive Technology Centre of a tertiary care hospital from December 2018 to June 2020. The research plan was accepted by the Institute Ethics Committee and was registered prospectively (CTRI/2018/12/016799). All participants provided written informed consent prior to recruitment. The study was carried out in accordance with the Helsinki declaration.

\section{Study population and participants}

Infertile women of age less than 38 years who have EMT persistently less than $7 \mathrm{~mm}$ on baseline endometrial evaluation before IVF despite standard HRT and hysteroscopically normal endometrial cavity and women with a history of cycle cancellation during FET cycles due to persistent thin endometrium were included in the study. All women with distorted endometrial cavity because of recurrent adhesions, submucous fibroids, endometrial polyps; severe endometriosis; adenomyosis and uterine anomalies were excluded from the study. EMT was measured at its thickest part in the sagittal plane of the uterus and was performed by the same clinician using a transvaginal probe of 5 to $9 \mathrm{MHz}$, on GE Voluson S6 (Voluson, GE Healthcare, India). Thin endometrium was defined as EMT $<7 \mathrm{~mm}$.

\section{Methodology (Fig. 1)}

In women with a history of cancelled FET cycles due to persistent thin endometrium, HRT in the form of estradiol valerate $6 \mathrm{mg} /$ day orally (Tab Progynova $2 \mathrm{mg}$; Zydus Cadila, India) starting from menstrual cycle day two was given to all the participants for endometrial preparation. Transvaginal ultrasound (TVS) was done on day 8. If EMT was less than $7 \mathrm{~mm}$, PRP was prepared and infused into the uterine cavity using an intrauterine (IUI) catheter. EMT and pattern were reassessed every 48 hours, and PRP infusion was repeated if required. When EMT reached more than $7 \mathrm{~mm}$, Injection progesterone $100 \mathrm{mg}$ intramuscular (Inj Susten $100 \mathrm{mg} / \mathrm{ml}$, Sun Pharma Laboratories Limited, India) daily was started, and embryo transfer was performed on day 3 or 5 accordingly.

In patients planned for fresh IVF cycles who were being evaluated for thin endometrium with a normal endometrial cavity in hysteroscopy, HRT in the form of estradiol valerate $6 \mathrm{mg} / \mathrm{d}$ orally (Tab Progynova $2 \mathrm{mg}$; Zydus Cadila, India) starting from menstrual cycle day 2 in an unstimulated (mock) cycle was given to all the participants for endometrial preparation. TVS was done on day 8. If EMT was less than $7 \mathrm{~mm}$, PRP was infused into the uterine cavity. EMT and pattern were reassessed every 48 hours, and PRP infusion was repeated if required. The patients were recruited in the present study for fresh IVF only when EMT reached $7 \mathrm{~mm}$. IVF was done according to the flexible GnRH antagonist protocol. During the stimulated cycle, if adequate EMT was obtained $(>7 \mathrm{~mm})$ during ovarian stimulation, the cycle was completed as per protocol, and no PRP was infused. If EMT was less than $7 \mathrm{~mm}$ on day 8 , intrauterine PRP was administered. EMT was reassessed after 48 hours, and PRP infusion was repeated if required. The maximum number of PRP infusions was limited to three. The cycle was completed with ovulatory trigger (hCG or dual) when 2-3 follicles achieved $18 \mathrm{~mm}$ diameter followed by oocyte retrieval after 34-36 hours. Embryo transfer was carried out on day 3 or 5 depending on embryo quality as assessed based on the Istanbul consensus (Alpha Scientists in Reproductive Medicine and ESHRE Special Interest Group of Embryology, 2011) only if EMT was more than $7 \mathrm{~mm}$ on the day of trigger.

Luteal phase support after embryo transfer was given with intramuscular injections of progesterone $100 \mathrm{mg} /$ day (Inj Susten, $100 \mathrm{mg} / \mathrm{ml}$, Sun Pharma Laboratories Limited, India). Serum $\beta$-hCG was measured two weeks after embryo transfer, and if positive, TVS was performed after another two weeks to confirm clinical pregnancy. Estradiol was also continued during the luteal phase until cardiac activity if pregnancy occurred.

\section{Method of autologous PRP preparation}

PRP was prepared from autologous blood under aseptic conditions using a two-step centrifuge process (Zadehmodarres et al., 2017). Fifteen millilitres of venous blood were drawn in a vial containing anticoagulant solution ACD-A (Anticoagulant Citrate Dextrose Solution, Solution A) and centrifuged immediately at $175 \mathrm{~g}$ for 12 minutes to separate the red blood cells. The plasma and buffy coat were collected in another empty tube and centrifuged again at $1300 \mathrm{~g}$ for 7 minutes. The resulting pellet of platelets was mixed with $1 \mathrm{ml}$ of supernatant and $0.5-1 \mathrm{ml}$ of PRP was instilled into the uterine cavity with an IUI catheter under ultrasound guidance within one hour of preparation. The technique of PRP preparation was standardised before the study to achieve platelet counts above $1,000,000 / \mu \mathrm{L}$.

\section{Primary and secondary outcomes of the study}

The primary endpoint was the expansion of EMT during fresh and FET cycle and according to the factor of infertility. The secondary outcomes were pregnancy rates (calculated per embryo transfer) in fresh and FET cycles and aetiology wise. Biochemical pregnancy was defined as serum $\beta$-hCG value of $\leq 100$ IU detected two weeks after embryo transfer and other failing values with no gestational sac. Clinical pregnancy was defined as evidence of pregnancy by visualisation of intrauterine gestational sac and foetal pole with a heartbeat on an ultrasound performed four weeks after embryo transfer. Implantation rate was defined as the ratio of the number of gestational sacs and the total number of embryos transferred. Live birth rate was defined as birth after 22 completed weeks of gestation with any evidence of life.

\section{Statistical Analysis}

Data was analysed using statistical software STATA version 14.0. Continuous variables were tested for normality using the Kolmogorov-Smirnov test. Descriptive 
Infertile women age less than 38 years assessed for eligibility criteria

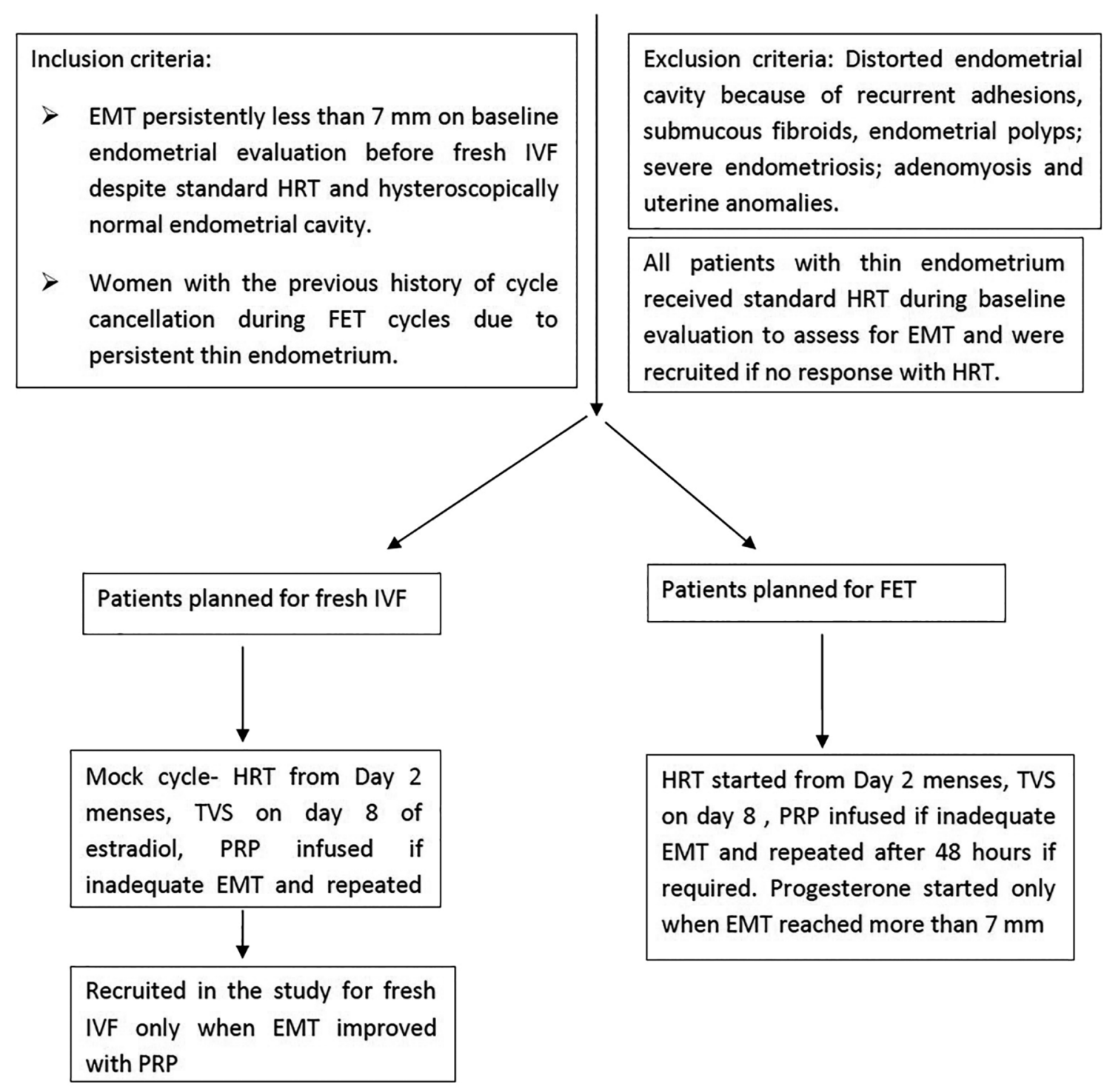

Figure 1. Algorithm depicting eligibility and recruitment criteria of participants in the study.

statistics such as mean, standard deviation and range values were calculated for normally distributed data. Comparison of mean values between the groups was tested using the independent t-test /ANOVA test as appropriate. Changes in measurement from before post-intervention within the group were compared using the paired t-test. For non-normal data, median and inter-quartile range values were calculated and compared using the non-parametric Mann-Whitney $U$ test. Categorical variables were presented as frequency and per cent values. Frequency data of categorical variables were compared using Chi-square/ Fisher's exact test as appropriate. For all statistical tests, a two-sided probability of $p$-value $<0.05$ was considered for statistical significance.

\section{RESULTS}

A total of 20 patients were enrolled in the study after assessing the eligibility criteria. The average age of the study population was $32.35 \pm 3.89$ years with mean BMI of $25.6 \pm 4.14 \mathrm{~kg} / \mathrm{m}^{2}$. The mean duration of infertility was $7.85 \pm 4.61$ years. The mean baseline EMT was $4.79 \pm 0.73 \mathrm{~mm}$ and mean EMT after oestrogen priming was $5.57 \pm 0.57 \mathrm{~mm}$. Basal FSH, LH, AMH and antral follicle count (AFC) are summarised in Table 1.

These women underwent 26 cycles of PRP, which were evaluated for an increase in EMT after PRP administration. Three patients did not undergo embryo transfer, and therefore only 23 cycles were assessed for pregnancy rates. One patient had empty follicle syndrome, a second patient had no live embryos after thawing, and a third patient with PCOS underwent a freeze all approach to prevent OHSS.

Out of 26 PRP cycles, 14 (53.8\%) were fresh IVF and 12 (46.2\%) were FET cycles. Tubal factor secondary to genital TB was present in $13(50.0 \%)$ cycles; PCOS was the cause of infertility in five $(19.2 \%)$ cycles whereas diminished ovarian reserve (DOR) was the underlying factor in eight $(30.8 \%)$ cycles. 
Table 1. Patient characteristics $(n=20)$.

\begin{tabular}{|l|c|}
\hline Parameters & Mean \pm SD \\
\hline Age $($ years $)$ & $32.35 \pm 3.89$ \\
\hline BMI $\left(\mathrm{kg} / \mathrm{m}^{2}\right)$ & $25.6 \pm 4.14$ \\
\hline Duration of infertility (years) & $7.85 \pm 4.61$ \\
\hline Basal FSH $(\mathrm{mIU} / \mathrm{l})$ & $5.74 \pm 1.66$ \\
\hline LH $(\mathrm{mIU} / \mathrm{l})$ & $4.4 \pm 1.86$ \\
\hline AMH $(\mathrm{ng} / \mathrm{ml})$ & $5.06 \pm 5.00$ \\
\hline AFC $(\mathrm{n})$ & $12.05 \pm 7.05$ \\
\hline
\end{tabular}

During fresh IVF, flexible antagonist protocol was used in all cycles. Recombinant hCG was used as a trigger in six cycles, and the dual trigger was given in the remaining eight cycles. The mean number of oocytes retrieved was $8.15 \pm 5.27$. Embryo transfer was done on either day 3 or 5 depending on embryo quality as assessed based on the Istanbul consensus. Mean number of embryos transferred was $2.4 \pm 0.67$.

All patients during FET cycles received the HRT protocol. Mean number of embryos transferred was 2.4 \pm 0.67 . Patients received no more than three infusions of PRP.

Comparison of primary and secondary outcomes in fresh vs. FET cycles (Table 2)

During Fresh IVF, mean \pm SD EMT before PRP was $5.83 \pm 0.81 \mathrm{~mm}$ and after PRP was $7.14 \pm 0.54 \mathrm{~mm}(p<0.001)$. After administration of the first dose of PRP, mean EMT rose significantly $(p<0.001)$ to $6.91 \pm 0.72 \mathrm{~mm}$. During FET cycles, mean EMT before PRP was $5.52 \pm 0.89 \mathrm{~mm}$ and after PRP was $7.14 \pm 0.68 \mathrm{~mm}(p<0.001)$. The first dose of PRP led to increase in EMT to $6.35 \pm 1.05 \mathrm{~mm}(p<0.001)$. The mean EMT before PRP, after PRP and average increase after first PRP, was comparable in fresh vs. FET cycles $(p>0.05)$. The endometrial pattern was triple layered during all PRP cycles.

The number of PRP infusions required was two in seven (50\%) cycles. EMT reached desired thickness with only one dose in the remaining seven $(50 \%)$ cycles during Fresh IVF whereas, in FET, eight cycles $(66.6 \%)$ required two doses, three cycles $(25 \%)$ needed one dose and only one cycle $(8.33 \%)$ required three doses to achieve the desired thickness. However, the number of PRP infusions required was statistically insignificant when fresh and FET cycles were compared $(p=0.313)$.

Two patients did not undergo embryo transfer in fresh IVF cycles. Clinical pregnancy (per transfer) was confirmed in $25 \%(3 / 12)$ of the patients; one of them had a twin gestation. There was no biochemical pregnancy. Implantation rate (IR) was $13.8 \%(4 / 29)$ and live birth rate (LBR) was $33.3 \%(4 / 12)$. Two patients underwent caesarean sections at term, and one had a preterm twin vaginal delivery.

During FET, one patient had no live embryos after thawing. Only two patients conceived, one had confirmed clinical pregnancy $(1 / 11 ; 9.1 \%)$, and another had a biochemical pregnancy. Implantation rate was $3.8 \%(1 / 26)$ with LBR of $9.1 \%(1 / 11)$. There was no significant $(p>0.05)$ difference between CPR, IR and LBR per transfer in fresh vs. FET cycles $(25 \%, 13.8 \%, 33.3 \%$ vs. $9.1 \%, 3.8 \%, 9.1 \%$, respectively).

None of the cycles was cancelled in fresh IVF or FET. There were no adverse reactions related to PRP administration.

Primary and secondary outcomes comparison by aetiology (Table 3 )

Tubal factor - Eleven patients underwent 13 PRP cycles during fresh IVF and FET. All women had laparoscopic evidence of TB with a history of intake of anti-tubercular treatment (ATT) for 6-9 months. Two patients had a history of hysteroscopic adhesiolysis as well. The mean EMT before PRP was $5.51 \pm 1.04 \mathrm{~mm}$ and after PRP was $7.15 \pm 0.61 \mathrm{~mm}$ $(p<0.001)$. Mean EMT rose to $6.55 \pm 0.87 \mathrm{~mm}$ after infusion of the first dose of PRP with a significant increase of $1.03 \mathrm{~mm}(p<0.001)$.

Diminished Ovarian reserve - Six patients underwent eight PRP cycles. The mean AMH was $1.82 \pm 1.1 \mathrm{ng} /$ $\mathrm{ml}$ with mean AFC of $7.83 \pm 2.8$. The mean EMT before PRP was $6.01 \pm 0.56 \mathrm{~mm}$ and after PRP was $7.42 \pm 0.44 \mathrm{~mm}$ $(p<0.001)$. There was a significant average increase of $1.05 \mathrm{~mm}$ in EMT after first PRP $(p=0.002)$.

PCOS - There were only three patients in this group who underwent five PRP cycles. The mean AMH and AFC were $11.8 \pm 4.6 \mathrm{ng} / \mathrm{ml}$ and $21.7 \pm 6.8$. The mean EMT before PRP was $5.62 \pm 0.61 \mathrm{~mm}$, after PRP $6.68 \pm 0.58 \mathrm{~mm}$ $(p<0.001)$. The average increase in EMT after first PRP was $0.64 \mathrm{~mm}(p=0.026)$.

There was no significant difference in mean EMT before PRP, after PRP or average increase after first PRP among the three groups $(p>0.05)$. The number of doses of PRP required to achieve the desired thickness was also comparable in the three aetiologies $(p=0.433)$ (Table 3 ).

Three patients conceived with no biochemical pregnancy in women with tubal factor infertility. Clinical pregnancy rate per transfer was $23.1 \%(3 / 13)$, and IR was $9.1 \%$ (3/33). All three patients had a caesarean section at term, yielding a LBR of $23.1 \%$ (3/13).

In the DOR group, one patient had a twin preterm vaginal delivery (CPR- $16.7 \%\{1 / 6\}$, IR- $16.7 \%\{2 / 12\}$ ), and another had a biochemical pregnancy. LBR was $33.3 \%$ $(2 / 6)$. None of the patients conceived in the PCOS group. There was no significant difference between CPR, IR and LBR among the three etiological factors $(p>0.05)$.

Comparison between conception vs. non-conception cycles (Table 4)

When cycles were divided into conception and non-conception subgroups, the number of fresh vs. FET cycles and the underlying etiological factor was comparable in both groups $(p>0.05)$. The baseline EMT was also comparable in women who conceived and women who did not $(p>0.714)$. The mean EMT before PRP was $5.6 \pm 0.62 \mathrm{~mm}$, and after PRP was $7.3 \pm 0.27 \mathrm{~mm}(p=0.010)$ in conception cycles, whereas in non-conception cycles mean EMT before PRP was $5.7 \pm 0.90 \mathrm{~mm}$ and after PRP was $7.1 \pm 0.65 \mathrm{~mm}(p<0.001)$. However, there was no significant difference in mean EMT before and after PRP in the two groups $(p>0.05)$.

\section{DISCUSSION}

Reports on clinical applications of PRP have vividly increased over the last decade. One cannot lay off the critical role played by platelet-derived growth factors on cell proliferation and neo-endothelial cell generation, which are chief elements in apt endometrial receptivity. In the 


\begin{tabular}{|c|c|c|c|}
\hline Outcome & Fresh IVF $(n=14)$ & FET $(n=12)$ & p-value \\
\hline EMT before PRP $(\mathrm{mm})$ & $5.83 \pm 0.81$ & $5.52 \pm 0.89$ & 0.349 \\
\hline EMT after PRP (mm) & $7.14 \pm 0.54$ & $7.14 \pm 0.68$ & 0.996 \\
\hline p-value & $<0.001$ & $<0.001$ & \\
\hline EMT after first PRP $(\mathrm{mm})$ & $6.91 \pm 0.72$ & $6.35 \pm 1.05$ & 0.123 \\
\hline Average increase after first PRP $(\mathrm{mm})$ & 1.07 & 0.83 & 0.267 \\
\hline$p$-value & $<0.001$ & $<0.001$ & \\
\hline $\begin{array}{l}\text { Number of PRP infusions } n(\%) \\
\text { One } \\
\text { Two } \\
\text { Three }\end{array}$ & $\begin{array}{l}7(50 \%) \\
7(50 \%) \\
0(0 \%)\end{array}$ & $\begin{array}{c}3(25 \%) \\
8(66.6 \%) \\
1(8.33 \%)\end{array}$ & 0.313 \\
\hline CPR per embryo transfer (\%) & $3 / 12(25 \%)$ & $1 / 11(9.1 \%)$ & 0.590 \\
\hline IR per embryo transfer $(\%)$ & $4 / 29(13.8 \%)$ & $1 / 26(3.8 \%)$ & 0.355 \\
\hline LBR per transfer (\%) & $4 / 12(33.3 \%)$ & $1 / 11(9.1 \%)$ & 0.317 \\
\hline
\end{tabular}

Data are mean \pm SD

CPR: Clinical pregnancy rate; IR: implantation rate; LBR: live birth rate

Table 3. Comparison of primary and secondary outcomes by aetiology types.

\begin{tabular}{|c|c|c|c|c|}
\hline & Tubal $(n=13)$ & DOR $(n=8)$ & $P \cos (n=5)$ & $p$-value \\
\hline EMT before PRP $(\mathrm{mm})$ & $5.51 \pm 1.04$ & $6.01 \pm 0.56$ & $5.62 \pm 0.61$ & 0.435 \\
\hline EMT after PRP $(\mathrm{mm})$ & $7.15 \pm 0.61$ & $7.42 \pm 0.44$ & $6.68 \pm 0.58$ & 0.086 \\
\hline $\mathrm{p}$-value & $<0.001$ & $<0.001$ & $<0.001$ & \\
\hline EMT after first PRP $(\mathrm{mm})$ & $6.55 \pm 0.87$ & $7.06 \pm 0.96$ & $6.26 \pm 0.86$ & 0.268 \\
\hline Average increase after first PRP ( $\mathrm{mm})$ & 1.03 & 1.05 & 0.64 & 0.340 \\
\hline $\mathrm{p}$-value & $<0.001$ & 0.002 & 0.026 & \\
\hline $\begin{array}{l}\text { No of PRP infusions } \mathrm{n}(\%) \\
\text { One } \\
\text { Two } \\
\text { Three }\end{array}$ & $\begin{array}{c}4(30.8 \%) \\
8(61.5 \%) \\
1(7.7 \%) \\
\end{array}$ & $\begin{array}{c}5(62.5 \%) \\
3(37.5 \%) \\
0(0 \%) \\
\end{array}$ & $\begin{array}{c}1(20 \%) \\
4(80 \%) \\
0(0 \%)\end{array}$ & 0.433 \\
\hline CPR per embryo transfer (\%) & $3 / 13(23.1 \%)$ & $1 / 6(16.7 \%)$ & $0 / 4(0 \%)$ & 0.789 \\
\hline IR per embryo transfer (\%) & $3 / 33(9.1 \%)$ & $2 / 12(16.7 \%)$ & $0 / 10(0 \%)$ & 0.553 \\
\hline LBR per transfer $(\%)$ & $3 / 13(23.1 \%)$ & $2 / 6(33.3 \%)$ & $0 / 4(0 \%)$ & 0.657 \\
\hline
\end{tabular}

Data are mean \pm SD

CPR, Clinical pregnancy rate; IR, implantation rate; LBR, live birth rate

\begin{tabular}{|l|c|c|c|}
\hline Table 4. Characteristics of conception vs. non-conception cycles. & $\begin{array}{c}\text { Non conception cycles } \\
\text { (n=21) }\end{array}$ & p-value \\
\hline Number of fresh vs. FET cycles & $\begin{array}{c}\text { Conception cycles } \\
(\mathbf{n = 5 )}\end{array}$ & $11(52.4 \%)$ & 0.995 \\
Fresh IVF, n (\%) & $3(60 \%)$ & $10(47.6 \%)$ & 0.660 \\
FET, n (\%) & $2(40 \%)$ & $10(47.6 \%)$ & $6(28.6 \%)$ \\
\hline Underlying aetiology & $3(60 \%)$ & $5(23.8 \%)$ & \\
Tubal factor, n (\%) & $2(40 \%)$ & & \\
DOR, n (\%) & $0(0 \%)$ & $4.81 \pm 0.69$ & 0.714 \\
PCOS, n (\%) & $4.94 \pm 0.72$ & $5.70 \pm 0.90$ & 0.817 \\
\hline EMT characteristics & $5.60 \pm 0.62$ & $7.11 \pm 0.65$ & 0.533 \\
Baseline EMT, mm & $7.28 \pm 0.27$ & $<0.001$ & \\
EMT before PRP, mm & 0.010 & & \\
EMT after PRP, mm & & & \\
$p$-value & & & \\
\hline
\end{tabular}

Data are mean \pm SD. 
present study, twenty women with persistent thin endometrium underwent 26 cycles of PRP. Fourteen (53.8\%) were fresh IVF and $12(46.2 \%)$ were FET cycles. Tubal factor secondary to genital TB was present in $13(50.0 \%)$ cycles; PCOS was the cause of infertility in five $(19.2 \%)$ cycles, whereas DOR was the underlying factor in eight $(30.8 \%)$ cycles.

To the extent of available facts, this is apparently the first study to report LBR after PRP administration in refractory thin endometrium in non-donor fresh IVF cycles. Also, LBR has been compared concerning the underlying factor of infertility. Mean EMT increased significantly after PRP administration during both fresh and FET cycles $(p<0.001)$. There was no significant $(p>0.05)$ difference between CPR, IR and LBR per transfer in fresh vs. FET cycles $(25 \%, 13.8 \%, 33.3 \%$ vs. $9.1 \%, 3.8 \%, 9.1 \%$, respectively).

LBR has been reported in only two studies to date. Molina et al. (2018) reported an LBR of $26.3 \%$ in 19 women with a history of refractory thin endometrium with at least one failed IVF attempt. However, this study included $31.6 \%$ donated oocyte cycles. LBR in non-donor cycles was $14.3 \%$. The present study included only non-donor cycles to remove the bias associated with proven high pregnancy rates in donor IVF cycles. Another study by Kim et al. (2019) reported an LBR of $20 \%$ in 20 women with refractory thin endometrium and two or more failed IVF attempts. However, they showed that there was no association between EMT changes and ET outcomes. Among the six clinical pregnancy cases, two had an increase, and four had a decrease in EMT. In both the studies, PRP was administered during HRT method of endometrial preparation during FET. The present study has shown an LBR of 33.1\% and $9.1 \%$ during fresh and FET cycles, respectively, with an overall LBR of $25 \%$. The difference in LBR in FET cycles may be attributed to small sample size in this group (only 12 cycles).

The primary outcome of EMT expansion after PRP administration during FET has been much explored in prior studies. EMT increased significantly in groups given PRP with significant improvement in implantation and clinical pregnancy rates (Zadehmodarres et al., 2017; Tandulwadkar et al., 2017; Eftekhar et al., 2018; Kim et al., 2019; Chang et al., 2019; Nazari et al., 2019). Cycle cessation rate was reduced in individuals administered PRP (Eftekhar et al., 2018; Chang et al., 2019) as compared to controls. A significant increase in vascularity was also noted following PRP administration by the number of vascular signals seen on Power Doppler, reaching zones 3 and 4 of the endometrium (Tandulwadkar et al., 2017). However, the endometrial and sub endometrial vascularity measured by 3D power Doppler ultrasound was found to be significantly lower in patients with low volume endometrium, but not in individuals with a thin endometrium $(\mathrm{Ng}$ et al., 2009). We did not assess endometrial and sub endometrial vascularity pre- and post PRP administration, as studies have described improved angiogenesis with PRP. Accordingly, the conclusions of this work are limited to only the thickness and pattern of the endometrium. The role of PRP in improving endometrial blood flow specific to aetiology needs to be explored in future studies. None of the studies has administered PRP in women having thin endometrium during ovarian stimulation.

The average increment in the EMT following PRP administration was $0.6 \mathrm{~mm}$, which was not found to be statistically significant (Kim et al., 2019). In our study, the average increase after the first dose of PRP was $0.83 \mathrm{~mm}$ in FET cycles and $1.07 \mathrm{~mm}$ during fresh IVF, which was statistically significant $(p<0.001)$. When compared aetiology wise, EMT increased significantly after the first PRP infusion by $1.03 \mathrm{~mm}, 1.05 \mathrm{~mm}, 0.64 \mathrm{~mm}$ in tubal, DOR and
PCOS women on average, respectively. No other study has documented the incremental expansion in EMT after one infusion of PRP, which is clinically relevant.

The procedure for preparing PRP is not yet standardised. Therefore, the ideal concentration of platelets and growth factors are not clearly defined (Amable et al., 2013). PRP with a platelet concentration of approximately $1,000,000 / \mu \mathrm{L}(503,000-1,729,000 / \mu \mathrm{L})$ is apparently needed to have a desirable effect. Whereas elevated concentrations might have paradoxically inhibitory results, the outcome is suboptimal with reduced concentrations (Weibrich et al., 2004). We standardised our technique of PRP preparation before starting recruiting the patients and used the double centrifuge method to have platelet counts above $1,000,000 / \mu \mathrm{L}$ for optimal effect.

There are four types of PRP preparations: leukocyte-poor or pure PRP (P-PRP), pure platelet-rich fibrin clot, leukocyte PRP (L-PRP) and leukocyte platelet-rich fibrin clot. The present study used the buffy coat of the centrifuge, i.e., L-PRP was employed as described in two previous studies (Chang et al., 2015; Zadehmodarres et al., 2017). The use of P-PRP has not yet been acknowledged in any of the studies.

The effectiveness of PRP specific to the underlying factor of infertility has not been explored in any study to date. We attempted to assess its role and whether it might be beneficial in a specific subset of the population with a thin endometrium. The endometrium is involved in $50-80 \%$ of women (Sharma et al., 2008) with tubal factor infertility secondary to genital TB leading to infertility. Eleven patients with tubal factor infertility underwent 13 PRP cycles during fresh IVF and FET in the present study. All women had laparoscopic evidence of TB with a history of intake of anti-tubercular treatment (ATT) for 6-9 months. Two patients had hysteroscopic adhesiolysis as well. EMT increased significantly after PRP administration in these poor prognosis patients with CPR and LBR of $23.1 \%$. Women with a diminished ovarian reserve may also be associated with thin endometrium, and PRP led to a significant increase in EMT with CPR and LBR of $16.7 \%$ and $33.3 \%$, respectively. Deferring fresh ET in these women based on thin endometrium may not be clinically possible sometimes because of the lower number of embryos available for cryopreservation. PRP has successfully been shown in the present study to increase the EMT during fresh IVF in these women to allow fresh ET. There were only three patients in the PCOS group who underwent five PRP cycles. Although the average increase in EMT after the first PRP infusion $(0.64 \mathrm{~mm})$ was significant, this increase was less as compared to other factors of infertility, although not significantly. No patient conceived in this group. These findings may have been affected by the small sample size in this subgroup and need to be explored in future studies. PCOS associated with hyperandrogenemia has been associated with a thin endometrium (Indhavivadhana et al., 2017). However, androgen levels were not measured in the present study. We could not elicit the cause of thin endometrium in these women. There was no significant difference in mean EMT before PRP, after PRP and average increase after first PRP infusion among the three groups $(p>0.05)$. The number of doses of PRP required to achieve the desired thickness was also comparable in all three aetiologies $(p=0.433)$. There was no significant difference between CPR, IR and LBR among three aetiological factors $(p>0.05)$. As none of the patients had unexplained infertility, our results cannot be extrapolated to this subgroup. More studies are required to explore the outcome of PRP in unexplained and other factors of infertility.

None of the cycles got cancelled during the study. No adverse effects were reported in our study, similar to Kim et al. (2019). PRP treatment, therefore, alleviates the 
frustration associated with repeated cycle cancellations associated with thin endometrium in these women. It is easily prepared, cost-effective, minimally invasive; rich in cytokines and growth factors, and carries minimal risk of infection transmission as autologous blood is used to prepare PRP.

\section{Strengths and weaknesses of the study}

This is the first study to report LBR after PRP administration in women with refractory thin endometrium who are considered to have poor prognosis for implantation and clinical pregnancy during fresh IVF-ET cycles. No study has assessed the effectiveness of PRP specific to underlying aetiology to date. We are the first to elucidate its role in thin endometrium of different pathophysiologies. PRP was prepared by a single lab technician, thus removing technical bias. All ultrasounds during study and PRP administration were performed entirely by the same clinician to allow for better standardisation of cycle management, making the study more robust clinically. Another major strength was our strict inclusion criteria of enrolling only women who responded to PRP during the mock cycle. Women who did not show any improvement in EMT expansion with PRP in an unstimulated cycle were excluded from the study. The mock cycle was conducted three months or more before the actual stimulation or FET cycle, thus removing the bias introduced because of endometrial scratch effect or the carry-over effect of PRP in subsequent cycles.

The major weakness was the small sample size, which limits its broader applicability. However, all the women included had thin endometrium refractory to standard treatments which nonetheless responded to PRP during the mock cycle. In clinical practice, we usually do not encounter such cases very frequently. Many women respond to the usual standard treatment protocols for endometrial preparation. Platelet counts were not measured during each PRP preparation before infusion. However, the method was standardised before recruiting the patients to have a desirable effect. We think the placebo or no treatment control arm was not justified for ethical reasons in these low prognosis group, and this study was intended to treat all patients with a refractory thin endometrium. Earlier randomised controlled trials offered absolutely conclusive encouraging evidence of PRP effectiveness. However, from a research point of view, more RCTs are required to evaluate the role of PRP in different pathophysiologies and fresh IVF cycles with thin endometrium during ovarian stimulation.

To conclude, the study findings imply that autologous PRP strives to increase the endometrial thickness significantly in both fresh and FET cycles in refractory thin endometrium associated with tubal factor secondary to genital TB, PCOS and DOR. The pregnancy outcomes in terms of clinical pregnancy and LBR reached up to $20 \%$ and $25 \%$, respectively, which is a noteworthy improvement in these low prognosis patients. PRP therapy offers an apposite option giving these patients a chance to achieve pregnancy by allowing embryo transfer which would not have been possible with standard treatments alone. The present study, therefore, favours the role of PRP in women who are deferred fresh ET or repeated cycle cancellations during FET because of their refractory thin endometrium. PRP should be administered to improve the reproductive outcome in the selected group of the population with a persistent thin endometrium.

Clinical Trial registration Number: CTRI/2018/12/016799
Acknowledgements: We thank Sangeeta Pant, Radiographer, ART Center, AIIMS, Delhi for helping during transvaginal ultrasound examinations and Bhuvan Bhaskar, Medical Lab Technologist, ART Center, AIIMS, Delhi for preparing PRP.

Funding: There was no support from any funding agency. The authors thank ART Center, Department of Obstetrics and Gynecology, AIIMS, Delhi to facilitate the conduct of the study.

\section{CONFLICT OF INTEREST}

None of the authors declare any conflict of interest.

\author{
Corresponding author: \\ Neeta Singh \\ ART Center \\ Department of Obstetrics \& Gynaecology \\ All India Institute of Medical Sciences \\ New Delhi, India \\ E-mail:drneetasingh@yahoo.com
}

\section{REFERENCES}

Aghajanova L, Houshdaran S, Balayan S, Manvelyan E, Irwin JC, Huddleston HG, Giudice LC. In vitro evidence that platelet-rich plasma stimulates cellular processes involved in endometrial regeneration. J Assist Reprod Genet. 2018;35:757-70 PMID: 29404863 DOI: 10.1007/s10815-018-1130-8

Amable PR, Carias RB, Teixeira MV, da Cruz Pacheco I, Corrêa do Amaral RJ, Granjeiro JM, Borojevic R. Platelet-rich plasma preparation for regenerative medicine: optimization and quantification of cytokines and growth factors. Stem Cell Res Ther. 2013;4:67. doi: 10.1186/scrt218 PMID: 23759113 DOI: $10.1186 /$ scrt218

Alpha Scientists in Reproductive Medicine and ESHRE Special Interest Group of Embryology. The Istanbul consensus workshop on embryo assessment: proceedings of an expert meeting. Hum Reprod. 2011;26:1270-83. PMID: 21502182 DOI: 10.1093/humrep/der037

Bu Z, Yang X, Song L, Kang B, Sun Y. The impact of endometrial thickness change after progesterone administration on pregnancy outcome in patients transferred with single frozen-thawed blastocyst. Reprod Biol Endocrinol. 2019;17:99. PMID: 31767010 DOI: 10.1186/s12958-019-0545-0

Cervelli V, Gentile P, Scioli MG, Grimaldi M, Casciani CU, Spagnoli LG, Orlandi A. Application of platelet-rich plasma in plastic surgery: clinical and in vitro evaluation. Tissue Eng Part C Methods. 2009;15:625-34. DOI: 10.1089/ten. TEC. 2008.0518 PMID: 19231923

Chang Y, Li J, Chen Y, Wei L, Yang X, Shi Y, Liang X. Autologous platelet-rich plasma promotes endometrial growth and improves pregnancy outcome during in vitro fertilisation. Int J Clin Exp Med. 2015;8:1286-90. PMID: 25785127 PMID: 25785127

Chang Y, Li J, Wei L, Pang J, Chen J, Liang X. Autologous platelet-rich plasma infusion improves clinical pregnancy rate in frozen embryo transfer cycles for women with thin endometrium. Medicine (Baltimore). 2019;98:e14062. PMID: 30653117 DOI: 10.1097/MD.0000000000014062 
Chen MJ, Yang JH, Peng FH, Chen SU, Ho HN, Yang YS. Extended estrogen administration for women with thin endometrium in frozen-thawed in-vitro fertilization programs. J Assist Reprod Genet. 2006;23:337-42. PMID: 16983519 DOI: $10.1007 / \mathrm{s} 10815-006-9053-1$

Eftekhar M, Neghab N, Naghshineh E, Khani P. Can autologous platelet rich plasma expand endometrial thickness and improve pregnancy rate during frozen-thawed embryo transfer cycle? A randomised clinical trial. Taiwan J Obstet Gynecol. 2018;57:810-3. PMID: 30545532 DOI: 10.1016/j.tjog.2018.10.007

Gargett CE, Chan RW, Schwab KE. Hormone and growth factor signaling in endometrial renewal: role of stem/progenitor cells. Mol Cell Endocrinol. 2008;288:22-9. DOI: 10.1016/j. mce.2008.02.026 PMID: 18403104

Gleicher N, Kim A, Michaeli T, Lee HJ, Shohat-Tal A, Lazzaroni $\mathrm{E}$, Barad $\mathrm{DH}$. A pilot cohort study of granulocyte colony-stimulating factor in the treatment of unresponsive thin endometrium resistant to standard therapies. Hum Reprod. 2013;28:172-7. PMID: 23081869 DOI: 10.1093/ humrep/des 370

Gonen Y, Casper RF. Prediction of implantation by the sonographic appearance of the endometrium during controlled ovarian stimulation for in vitro fertilisation (IVF). J Vitro Fert Embryo Transf. 1990;7:146-52. PMID: 2199588 DOI: $10.1007 /$ BF01135678

Hadi FH, Chantler E, Anderson E, Nicholson R, McClelland RA, Seif MW. Implantation: ovulation induction and endometrial steroid receptors. Hum Reprod. 1994;9:2405-10. DOI: $10.1093 /$ oxfordjournals.humrep.a138459

Hsu CC, Kuo HC, Wang ST, Huang KE. Interference with uterine blood flow by clomiphene citrate in women with unexplained infertility. Obstet Gynecol. 1995;86:917-21. PMID: 7501339 DOI: 10.1016/0029-7844(95)00287-2

Indhavivadhana $S$, Rattanachaiyanont $M$, Wongwananuruk T, Techatraisak K, Tanmahasamut $P$, Dangrat C. Brief communication (Original). Hyperandrogenemia is associated with thin endometrium in reproductive-aged Thai women with polycystic ovary syndrome. Asian Biomed. 2017;7:545-51. DOI: 10.5372/1905-7415.0704.210

Jo $\mathrm{CH}$, Shin JS, Lee YG, Shin WH, Kim H, Lee SY, Yoon KS, Shin S. Platelet-rich plasma for arthroscopic repair of large to massive rotator cuff tears: a randomized, single-blind, parallel-group trial. Am J Sports Med. 2013;41:2240-8. PMID: 23921338 DOI: 10.1177/0363546513497925

Kasius A, Smit JG, Torrance HL, Eijkemans MJ, Mol BW, Opmeer BC, Broekmans FJ. Endometrial thickness and pregnancy rates after IVF: a systematic review and meta-analysis. Hum Reprod Update. 2014;20:530-41. PMID: 24664156 DOI: 10.1093/humupd/dmu011

Kim H, Shin JE, Koo HS, Kwon H, Choi DH, Kim JH. Effect of Autologous Platelet-Rich Plasma Treatment on Refractory Thin Endometrium During the Frozen Embryo Transfer Cycle: A Pilot Study. Front Endocrinol (Lausanne). 2019;10:61. PMID: 30837945 DOI: 10.3389/fendo.2019.00061
Liu KE, Hartman M, Hartman A. Management of thin endometrium in assisted reproduction: a clinical practice guideline from the Canadian Fertility and Andrology Society. Reprod Biomed Online. 2019;39:49-62. doi: 10.1016/j. rbmo.2019.02.01 PMID: 31029557 DOI: $10.1016 / \mathrm{j}$. rbmo.2019.02.013

Mahajan N, Sharma S. The endometrium in assisted reproductive technology: How thin is thin? J Hum Reprod Sci. 2016;9:3-8. PMID: 27110071 DOI: 10.4103/09741208.178632

Matsumoto $H$, Nasu K, Nishida M, Ito H, Bing S, Miyakawa I. Regulation of proliferation, motility, and contractility of human endometrial stromal cells by platelet-derived growth factor. J Clin Endocrinol Metab. 2005;90:35607. DOI: $10.1210 /$ jc. 2004-1918 PMID: 15755859 DOI: 10.1210/jc. $2004-1918$

Molina A, Sánchez J, Sánchez W, Vielma V. Platelet-rich plasma as an adjuvant in the endometrial preparation of patients with refractory endometrium. JBRA Assist Reprod. 2018;22:42-8. PMID: 29303234 DOI: 10.5935/15180557.20180009

Nazari L, Salehpour S, Hoseini S, Zadehmodarres S, Azargashb E. Effects of autologous platelet-rich plasma on endometrial expansion in patients undergoing frozen-thawed embryo transfer: A double-blind RCT. Int J Reprod Biomed. 2019;17:443-8. PMID: 31508569 DOI: 10.18502/ijrm.v17i6.4816

$\mathrm{Ng} \mathrm{EH}$, Yeung WS, Ho PC. Endometrial and subendometrial vascularity are significantly lower in patients with endometrial volume $2.5 \mathrm{ml}$ or less. Reprod Biomed Online. 2009;18:262-8. PMID: 19192348 DOI: 10.1016/S14726483(10)60264-7

Paulson RJ, Sauer MV, Lobo RA. Embryo implantation after human in vitro fertilisation: importance of endometrial receptivity. Fertil Steril. 1990;53:870-4. PMID: 2110078 DOI: $10.1016 / S 0015-0282(16) 53524-9$

Picard F, Hersant B, Bosc R, Meningaud JP. The growing evidence for the use of platelet-rich plasma on diabetic chronic wounds: A review and a proposal for a new standard care. Wound Repair Regen. 2015;23:638-43. PMID: 26019054 DOI: 10.1111/wrr.12317

Scheffer GJ, Broekmans FJ, Looman CW, Blankenstein M, Fauser $\mathrm{BC}$, teJong $\mathrm{FH}$, teVelde $\mathrm{ER}$. The number of antral follicles in normal women with proven fertility is the best reflection of reproductive age. Hum Reprod. 2003;18:7006. PMID: 12660259 DOI: 10.1093/humrep/deg135

Sharma JB, Roy KK, Pushparaj M, Gupta N, Jain SK, Malhotra N, Mittal S. Genital tuberculosis: an important cause of Asherman's syndrome in India. Arch Gynecol Obstet. 2008;277:37-41. doi: 10.1007/s00404-007-0419-0 PMID: 17653564 DOI: $10.1007 / \mathrm{s} 00404-007-0419-0$

Sher G, Fisch JD. Effect of vaginal sildenafil on the outcome of in vitro fertilisation (IVF) after multiple IVF failures attributed to poor endometrial development. Fertil Steril. 2002;78:1073-6. PMID: 12413996 DOI: 10.1016/S00150282(02)03375-7 
Tandulwadkar SR, Naralkar MV, Surana AD, Selvakarthick $\mathrm{M}$, Kharat $\mathrm{AH}$. Autologous Intrauterine Platelet-Rich Plasma Instillation for Suboptimal Endometrium in Frozen Embryo Transfer Cycles: A Pilot Study. J Hum Reprod Sci. 2017;10:208-12. PMID: 29142450 DOI: 10.4103/jhrs. JHRS_28_17

Thessaloniki ESHRE/ASRM-Sponsored PCOS Consensus Workshop Group. Consensus on infertility treatment related to polycystic ovary syndrome. Hum Reprod. 2008;23:46277. PMID: 18308833 DOI: 10.1093/humrep/dem426

von Wolff M, Fäh M, Roumet M, Mitter V, Stute $P$, Griesinger G, Kohl Schwartz A. Thin Endometrium Is Also Associated With Lower Clinical Pregnancy Rate in Unstimulated Menstrual Cycles: A Study Based on Natural Cycle IVF. Front Endocrinol. 2018;9:776. PMID: 30619099 DOI: 10.3389/ fendo.2018.00776

Weckstein LN, Jacobson A, Galen D, Hampton K, Hammel J. Low-dose aspirin for oocyte donation recipients with a thin endometrium: prospective, randomized study. Fertil Steril. 1997;68:927-30. PMID: 9389827 DOI: 10.1016/ S0015-0282(97)00330-0
Weibrich G, Hansen T, Kleis W, Buch R, Hitzler WE. Effect of platelet concentration in platelet-rich plasma on peri-implant bone regeneration. Bone. 2004;34:665-71. PMID: 15050897 DOI: $10.1016 /$ j.bone.2003.12.010

Zadehmodarres S, Salehpour S, Saharkhiz N, Nazari $L$. Treatment of thin endometrium with autologous platelet-rich plasma: a pilot study. BRA Assist Reprod. 2017;21:54-6. PMID: 28333034 DOI: 10.5935/15180557.20170013

Zhang T, Li Z, Ren X, Huang B, Zhu G, Yang W, Jin L. Endometrial thickness as a predictor of the reproductive outcomes in fresh and frozen embryo transfer cycles: A retrospective cohort study of 1512 IVF cycles with morphologically good-quality blastocyst. Medicine. 2018;97:e9689. PMID: 29369190 DOI: $10.1097 / M D .0000000000009689$

Zhao J, Zhang Q, Wang Y, Li Y. Endometrial pattern, thickness and growth in predicting pregnancy outcome following 3319 IVF cycle. Reprod Biomed Online. 2014;29:291-8. PMID: 25070912 DOI: 10.1097/MD.0000000000009689 